\title{
KLOE extraction of Vus from kaon decays and lifetimes
}

\section{KLOE collaboration *}

presented by Barbara Sciascia ${ }^{\dagger}$

INFN Frascati, Italy

E-mail: barbara.sciascia@lnf.infn.it

The most precise determinations of $V_{\mathrm{us}}$ comes from semileptonic kaon decays. The KLOE experiment at DAФNE the Frascati $\phi$ factory, has measured all the experimental inputs to $V_{\mathrm{us}}$ for both neutral and charged kaons. Preliminary results for $B R\left(K^{ \pm} \rightarrow \pi^{0} e^{ \pm} v\right)$ and $B R\left(K^{ \pm} \rightarrow \pi^{0} \mu^{ \pm} v\right)$, and for $\tau_{ \pm}$are presented, together with measurements of the $K_{L} e 3$ and $K_{L} \mu 3$ decay BR, the $K_{S} e 3$ decay BR and the $K_{L}$ lifetime $\tau_{L}$. From our results for the 5 branching ratios and $\tau_{L}$ we find $V_{\mathrm{us}}=0.2258 \pm 0.0022$. We have also measured the fully inclusive $K_{\mu 2}^{+}(\gamma)$ absolute branching ratio for which we obtain $\mathrm{BR}\left(K^{+} \rightarrow \mu v(\gamma)\right)=0.6366 \pm 0.0017$. Combining this value with recent lattice results for $f_{K} / f_{\pi}$ gives $V_{\mathrm{us}}=0.2223 \pm 0.0026$.

International Europhysics Conference on High Energy Physics

July 21st - 27th 2005

Lisboa, Portugal

${ }^{*}$ F. Ambrosino, A. Antonelli, M. Antonelli, C. Bacci, P. Beltrame, G. Bencivenni, S. Bertolucci, C. Bini, C. Bloise, V. Bocci, F. Bossi, D. Bowring, P. Branchini, R. Caloi, P. Campana, G. Capon, T. Capussela, F. Ceradini, S. Chi, G. Chiefari, P. Ciambrone, S. Conetti, E. De Lucia, A. De Santis, P. De Simone, G. De Zorzi, S. Dell'Agnello, A. Denig, A. Di Domenico, C. Di Donato, S. Di Falco, B. Di Micco, A. Doria, M. Dreucci, G. Felici, A. Ferrari, M. L. Ferrer, G. Finocchiaro, C. Forti, P. Franzini, C. Gatti, P. Gauzzi, S. Giovannella, E. Gorini, E. Graziani, M. Incagli, W. Kluge, V. Kulikov, F. Lacava, G. Lanfranchi, J. Lee-Franzini, D. Leone, M. Martini, P. Massarotti, W. Mei, L. Meola, S. Miscetti, M. Moulson, S. Müller, F. Murtas, M. Napolitano, F. Nguyen, M. Palutan, E. Pasqualucci, A. Passeri, V. Patera, F. Perfetto, L. Pontecorvo, M. Primavera, P. Santangelo, E. Santovetti, G. Saracino, B. Sciascia, A. Sciubba, F. Scuri, I. Sfiligoi, A. Sibidanov, T. Spadaro, M. Testa, L. Tortora, P. Valente, B. Valeriani, G. Venanzoni, S. Veneziano, A. Ventura, R. Versaci, G. Xu.

'Speaker. 
The most precise verification of the unitarity of the CKM mixing matrix is obtained today from the $V_{\text {us }}$ and $V_{\text {ud }}$ values, neglecting $\left|V_{\text {ub }}\right|^{2} \sim 0.00002$. With the KLOE detector we can measure all experimental inputs to $V_{\mathrm{us}}$ : branching ratios, lifetimes, and form factors.

\section{DA $\Phi N E$ and KLOE}

In the DAФNE $e^{+} e^{-}$collider, beams collide at a center-of-mass energy $W \sim M(\phi)$. Since 2001, KLOE has collected an integrated luminosity of $\sim 2 \mathrm{fb}^{-1}$. Results presented below are based on 2001-02 data for $\sim 450 \mathrm{pb}^{-1}$.

The KLOE detector consists of a large cylindrical drift chamber surrounded by a lead/scintillating-fiber electromagnetic calorimeter. A superconducting coil around the calorimeter provides a $0.52 \mathrm{~T}$ field. The drift chamber, Ref. [1], is $4 \mathrm{~m}$ in diameter and $3.3 \mathrm{~m}$ long. The momentum resolution is $\sigma\left(p_{T}\right) / p_{T} \sim 0.4 \%$. Two track vertices are reconstructed with a spatial resolution of $\sim 3 \mathrm{~mm}$. The calorimeter, Ref. [2], composed of a barrel and two endcaps, covers $98 \%$ of the solid angle. Energy and time resolution are $\sigma(E) / E=5.7 \% / \sqrt{E(\mathrm{GeV})}$ and $\sigma(t)=54 \mathrm{ps} / \sqrt{E(\mathrm{GeV})} \oplus$ 50 ps. The KLOE trigger, Ref. [3], uses calorimeter and drift chamber information. For the present analysis only the calorimeter signals are used. Two energy deposits above threshold, $E>50 \mathrm{MeV}$ for the barrel and $E>150 \mathrm{MeV}$ for the endcaps, are required.

\section{Kaon tagging}

The $\phi$ meson decays mainly into kaons: $49 \%$ to $K^{+} K^{-}$and $34 \%$ to $K_{L^{-}} K_{S}$ pairs. We can thus $\operatorname{tag} K_{L}, K_{S}, K^{+}$, and $K^{-}$beams by detecting respectively $K_{S}, K_{L}, K^{-}$, and $K^{+}$decays. Tagging allows measurements of absolute branching ratios. A $K_{S}$ beam is tagged using events with a $K_{L}$ interaction in the calorimeter. KLOE results on $K_{S}$ decays are given in these proceedings [4]. $K_{L^{-}}$-mesons are tagged detecting $K_{S} \rightarrow \pi^{+} \pi^{-}$decays. Charged kaons are tagged using two-body decays, $K^{ \pm} \rightarrow \mu^{ \pm} v$ and $K^{ \pm} \rightarrow \pi^{ \pm} \pi^{0}$. Perfect tagging requires that the detection efficiency of the tagging mode be independent of the decay mode of the tagged kaon. In reality, some dependency of the tagging efficiency on the decay mode of the signal kaon exists. This dependence must be carefully measured using Monte Carlo (MC) and data control samples for each BR measurement.

\section{Semileptonic $K^{ \pm}$decays}

The measurement of the branching ratios for the $K^{ \pm}$semileptonic decays is performed using four samples defined by different decay modes for the tagging kaon: $K_{\mu 2}^{+}, K_{\pi 2}^{+}, K_{\mu 2}^{-}$, and $K_{\pi 2}^{-}$. This redundancy allows the systematic effects due to the tag selection to be kept under control. Kaons are identified as a tracks with momentum $70<p<130 \mathrm{MeV}$, originating from the collision point. The kaon decay vertex must be within a fiducial volume (FV) defined as a cylinder of radius $40<r<150 \mathrm{~cm}$, centered at the collision point, coaxial with the beams. The decay track, extrapolated to the calorimeter, must overlap an appropriate energy deposit. $K_{\mu 2}$ and $K_{\pi 2}$ decays are selected by applying a $3 \sigma$ cuts around the kaon rest-frame momentum distribution calculated in the relevant mass hypothesis. For the $K_{\pi 2}^{ \pm}$tag, identification of the $\pi^{0}$ from the vertex is also required. Finally, to reduce the dependency of the tag selection efficiency on the decay mode of the 
signal kaon, the tagging decay is required to satisfy the calorimeter trigger. In the analyzed data set about 60 million tag decays were identified and divided into the four tag samples. To select a semileptonic decay on the signal side, a one-prong kaon decay vertex must be present in the FV. The daughter track has to reach the calorimeter and to overlap an energy deposit. Two-body decays are rejected by requiring that the decay momentum in the kaon frame, computed assuming the pion mass, is $<195 \mathrm{MeV}$. The lepton mass is obtained from the velocity of the lepton computed from the time of flight. The number of $K_{e 3}$ and $K_{\mu 3}$ decays is then obtained by fitting the $m_{\text {lept }}^{2}$ distribution to a sum of MC distributions for the signals and various background sources, each multiplied by a free scale factor. An example of the lepton mass distribution is shown in fig. 1, left. The BR
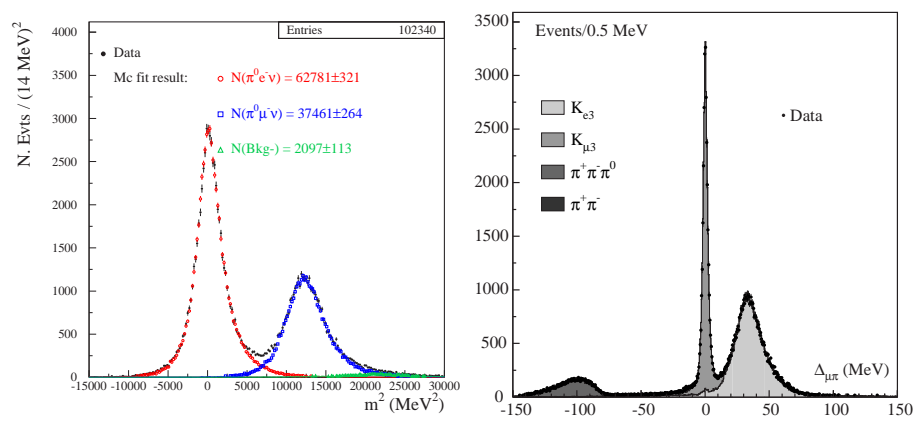

Figure 1: Left. Lepton mass distribution. Right. $\Delta_{\mu \pi}$ distribution.

is evaluated separately for each tag sample, dividing by the number of tag counts and correcting for acceptances. The latter are obtained from MC simulations. Corrections are applied to account for data-MC differences in tracking and cluster. About $190000 K_{e 3}^{ \pm}$and $100000 K_{\mu 3}^{ \pm}$decays are selected. The resulting BR's are:

$$
\begin{aligned}
& B R\left(K_{e 3}^{ \pm}\right)=\left(5.047 \pm 0.046_{\text {Stat }+ \text { Tag }} \pm 0.080_{\text {Syst }}\right) \% \\
& B R\left(K_{\mu 3}^{ \pm}\right)=\left(3.310 \pm 0.040_{\text {Stat }+ \text { Tag }} \pm 0.070_{\text {Syst }}\right) \% .
\end{aligned}
$$

These values are averages over the four different tag samples for each channel, and have been calculated with correlations carefully taken into account. The error is dominated by the error on data/MC efficiency corrections and the systematic error from the signal selection efficiency is still preliminary.

\section{4. $K^{ \pm}$lifetime}

The $K^{ \pm}$lifetime is an experimental input to the determination of $V_{\text {us. }}$ The present fractional uncertainty is about $0.2 \%$, corresponding to an uncertainty of $0.1 \%$ for $V_{\text {us }}$. However, see Ref. [7], there are large discrepancies between results of different experiments. The value of $\tau_{ \pm}$also affects the geometrical acceptance in BR measurements. KLOE is nearing completion of a new, highstatistics $\tau_{ \pm}$measurement using two different methods: one based on the measurement of the decay length and the other on the decay time of the kaons. Both methods have fractional errors at the few per mil level; comparison between the methods allows part of the systematic error to be assessed. 


\section{Semileptonic $K_{L}$ decays and the $K_{L}$ lifetime}

KLOE has also measured the dominant $K_{L}$ branching ratios using the $K_{L}$ beam tagged by $K_{S} \rightarrow \pi^{+} \pi^{-}$decays [6]. About $13 \times 10^{6}$ tagged $K_{L}$ decays are used for the measurement, and $\sim 4 \times 10^{6}$ to evaluate efficiencies. For $K_{e 3}, K_{\mu 3}$, and $\pi^{+} \pi^{-} \pi^{0}$ decays we compute smallest of the two values of $\Delta=E_{\text {miss }}-p_{\text {miss }}$ assuming each charged particle to be pion and muon (muon and pion). The distribution in this variable shows three well separated peaks corresponding to the three modes mentioned, see Fig. 1, right. Fitting the distribution with MC obtained shapes for each mode, gives the number of events in each channel. To select $K_{L} \rightarrow 3 \pi^{0}$ events, at least three photons are required from the $K_{L}$ decay vertex. The reconstruction efficiency and purity of the selected sample are both about $99 \%$.

The resulting BR's are: $\mathrm{BR}\left(K_{L} \rightarrow \pi e v(\gamma)\right)=0.4007 \pm 0.0006 \pm 0.0014_{T a g+T r k}, \mathrm{BR}\left(K_{L} \rightarrow\right.$ $\pi \mu v(\gamma))=0.2698 \pm 0.0006 \pm 0.0014_{\text {Tag }+ \text { Trk }}, \mathrm{BR}\left(K_{L} \rightarrow 3 \pi^{0}\right)=0.1997 \pm 0.0005 \pm 0.0019_{\text {Tag }+\gamma_{\text {count }}}$ and, $\operatorname{BR}\left(K_{L} \rightarrow \pi^{+} \pi^{-} \pi^{0}(\gamma)\right)=0.1263 \pm 0.0005 \pm 0.0011_{\text {Tag }+T r k}$, after imposing the constraint $\sum \operatorname{BR}\left(K_{L}\right)=1$. This corresponds to also measuring the lifetime by counting the number of decays in a time interval for a beam of known intensity.

The $K_{L}$ lifetime has been also measured directly [8], employing $10^{7} K_{L} \rightarrow 3 \pi^{0}$ events. The result is $\tau_{L}=(50.92 \pm 0.17 \pm 0.25) \mathrm{ns}$, which together with that from the $K_{L}$ BR measurements gives the KLOE average: $\tau_{L}=(50.84 \pm 0.23) \mathrm{ns}$

\section{Determination of $V_{\mathrm{us}}$}

The BR's of the semileptonic $K_{L}$ decays, together with the result $B R\left(K_{S} \rightarrow \pi e v(\gamma)\right)=(7.09 \pm$ $0.09) \times 10^{-4}$ and the preliminary results on the semileptonic $K^{ \pm}$decays, allow five independent determinations of the observable $\left|V_{\text {us }} f_{+}(0)\right|$, as shown in Fig. 2, in which the new KLOE value of $\tau_{L}$ has been used to covert $K_{L}$ BR's to partial widths. Averaging the five KLOE values gives $\left|V_{\text {us }} f_{+}(0)\right|=0.2170 \pm 0.0005$, with $\chi^{2} / \mathrm{DoF}=1.7 / 4$. The fractional uncertainty is about $0.25 \%$.

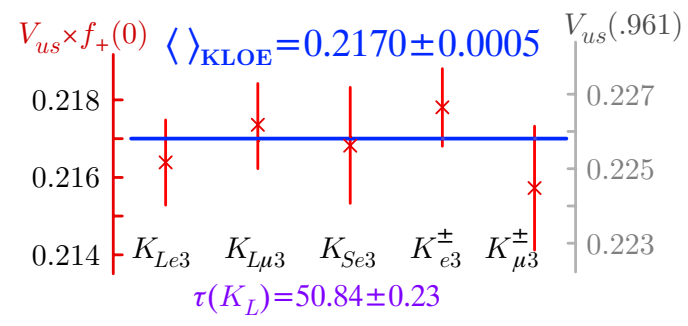

Figure 2: $\left|V_{\mathrm{us}} f_{+}(0)\right|$ measurements. For $K_{L}$ BR the KLOE $\tau_{L}$ has been used.

A precise estimate of $f_{+}(0), 0.961 \pm 0.008$, was first given in 1984 [9]. Recent lattice calculations [10] give $f_{+}(0)=0.960 \pm 0.009$, in agreement with Ref. [9]. Using the value from [9] and the average of our results for $\left|V_{\mathrm{us}} f_{+}(0)\right|$ we find $V_{\mathrm{us}}=0.2258 \pm 0.0022$.

7. $V_{\mathrm{us}}$ from $B R\left(K^{+} \rightarrow \mu^{+} v(\gamma)\right)$

KLOE has also measured the fully inclusive, absolute $K_{\mu 2}^{+}$branching ratio. From about $9 \times 10^{5}$ 
$K_{\mu 2}^{+}$decays obtained from a sample of about $250 \mathrm{pb}^{-1} \mathrm{KLOE}$ obtains $B R\left(K^{+} \rightarrow \mu^{+} v(\gamma)\right)=0.6366 \pm 0.0009_{\text {Stat }}$ $\pm 0.0015_{\text {Syst }}[11]$, for an overall fractional error of $0.27 \%$. Using recent lattice results on the decay constants of pseudoscalar mesons [5], we find $V_{\mathrm{us}}=0.2223 \pm 0.0026$. The fractional error of about $1 \%$ is dominated by the uncertainty in the $f_{K} / f_{\pi}$ computation.

\section{References}

[1] M. Adinolfi et al., [KLOE Collaboration], The tracking detector of the KLOE experiment, Nucl. Instrum. Meth A 488200251

[2] M. Adinolfi et al., [KLOE Collaboration], The KLOE electomagnetic calorimeter, Nucl. Instrum. Meth A 4822002364

[3] M. Adinolfi et al., [KLOE Collaboration], The trigger system of the KLOE experiment, Nucl. Instrum. Meth A 4922002134

[4] M. Moulson, KLOE results on neutral kaon decays and searches, these proceedings

[5] C. Aubin et al., MILC Collaboration, Phys. Rev. D 702004114501 W.J. Marciano, Phys. Rev. Lett. 932004231803

[6] F. Ambrosino et al., [KLOE Collaboration], Phys. Lett. B, in print, hep-ex/0508027

[7] S. Eidelman et al., Particle Data Group, Phys. Lett. B 59220041

[8] F. Ambrosino et al., [KLOE Collaboration], Phys. Lett. B 6262005 15-23

[9] H. Leutwyler and M. Roos, Z. Phys. C25 198491

[10] D. Becirevic et al., Nucl. Phys. B 7052005339

[11] F. Ambrosino et al., [KLOE Collaboration], Phys. Lett. B, in print, hep-ex/0509045 